\title{
ECTD Supplement Effective Date Type Terminology
}

National Cancer Institute

\section{Source}

National Cancer Institute. eCTD Supplement Effective Date Type Terminology. NCI

Thesaurus. Code C97191.

Terminology developed to support eCT D Supplemental Effect Date Types. 